\title{
EXPERIENCIAS DE AGRICULTURA SUSTENTABLE Y COMERCIO JUSTO EN EL ESTADO DE JALISCO, OCCIDENTE DE MÉXICO
}

\author{
Peter R. W. Gerritsen \\ petergerritsen@cucsur.udg.mx \\ DEPARTAMENTO DE ECOLOGÍA Y RECURSOS NATURALES, IMECBIO \\ CENTRO UNIVERSITARIO DE LA COSTA SUR \\ UNIVERSIDAD DE GUADALAJARA \\ Jaime Morales Hernández \\ jaimem@iteso.mx \\ CENTRO DE INVESTIGACIÓN Y FORMACIÓN SOCIAL \\ INSTITUTO TECNOLÓGICO DE ESTUDIOS SUPERIORES DE OCCIDENTE \\ TLAQUEPAQUE, JALISCO
}

\section{RESUMEN}

En este artículo se describen las experiencias de diferentes grupos de productores en el estado de Jalisco y sus esfuerzos para desarrollar un modelo de desarrollo alternativo. Desde hace varios años, han trasformado sus sistemas de producción convencionales en sustentables, asimismo han buscado canales de comercialización diferentes. Los grupos tienen en común su participación en la Red de Alternativas Sustentables Agropecuarias — RASA—, donde también participan diferentes instituciones no gubernamentales y grupos de consumidores. Esta red se puede considerar como un nuevo movimiento social que busca desarrollar nuevos esquemas de desarrollo rural sustentable. Terminamos con una discusión y conclusión en relación con las posibilidades y desafíos de la Red para fortalecer la agricultura sustentable y el comercio justo.

Palabras clave: Agricultura sustentable, comercio justo, movimientos sociales. 


\section{ABSTRACT}

This article describes the experiences of different groups of producers in the state of Jalisco and their efforts to develop an alternative development model. Beginning several years ago, they have transformed their conventional production systems into sustainable systems, and have also sought different commercialization channels. The groups have in common their participation in the Network of Sustainable Agricultural Alternatives (Red de Alternativas Sustentables Agropecuarias -RASA) in which different nongovernmental institutions and consumer groups also participate. This network may be considered a new social movement that aims to develop new schemes of sustainable rural development. We end with a discussion and conclusion in relation with the possibilities and challenges of the Network to strengthen sustainable agriculture and fair trade.

Key words: Sustainable agriculture, fair trade, social movements. 


\section{INTRODUCCIÓN}

La descentralización es actualmente una política común en muchos países alrededor del mundo. Es comprendida como la trasferencia de poder y recursos de la agencia central a agencias periféricas. La descentralización puede tomar diversas formas y comúnmente es confundida con la desconcentración, siendo esta última la trasferencia de tareas administrativas a autoridades de menor nivel (Fraga 2000).

La descentralización y la desconcentración tienen como objetivo reducir la distancia política entre el Estado y sus ciudadanos. Ambos procesos no pueden ser comprendidos sin contar con la globalización, especialmente en su dimensión económica. La globalización ha sido conceptualizada principalmente en términos neoliberales $\mathrm{y}$, como tal, puede ser considerada como una nueva fase en la expansión del capitalismo occidental (Arrighi 1999). Es también dentro de este mismo contexto que han surgido muchos movimientos de ámbito local (Beck 1992, Morales 2004). Comúnmente, estos movimientos se han beneficiado de los nuevos espacios políticos creados como parte de los procesos de descentralización.

La investigación acerca de la descentralización se ha enfocado en diferentes aspectos: sus ventajas políticas y económicas, su impacto sobre el desarrollo rural, el desempeño de los servicios públicos, la solución a la pobreza, el remedio a la crisis fiscal, la estabilidad política y macroeconómica, la unidad nacional y edificación del Estado, o el incremento en la legitimidad del gobierno (Larson y Ribbot 2004). Impulsar la descentralización se considera difícil, y se puede decir que sus avances todavía no son apreciables, aun cuando existen avances importantes en muchos países. Además, un 
prerrequisito para una descentralización exitosa es la capacidad de los actores locales de movilizar mayores demandas a las autoridades, que no siempre se da (ibíd.).

En este escrito nos acercaremos a un incipiente movimiento social en el occidente de México relacionado con la agricultura sustentable y el comercio justo. Este movimiento se llama Red de Alternativas y Agropecuarias de Jalisco — referida como RASA—, el cual puede ser considerado como una iniciativa local cuyo objetivo es fortalecer el desarrollo rural sustentable. El objetivo principal de RASA es la revalorización de las relaciones campo-ciudad, y sus actividades de comercio justo tienen que ser entendidas en este sentido. Más que dirigirse a los mercados de los países del norte, tiene que ver con la creación de nichos nuevos en los mercados locales.

A continuación presentamos algunas reflexiones teóricas sobre la descentralización, movimientos sociales y manejo de recursos naturales, así como una descripción de la problemática actual en el campo mexicano; después nos enfocamos en la experiencia de RASA. Nuestro escrito termina con una discusión y conclusión, contextualizadas en las discusiones sobre descentralización, movimientos sociales y manejo de recursos naturales.

\section{DESCENTRALIZACIÓN Y MANEJO DE RECURSOS NATURALES}

La descentralización se ha dado también en relación con el uso y manejo de los recursos naturales. En este ámbito se pueden observar tensiones entre los recursos, concebidos como fuentes de subsistencia o como base para el desarrollo económico nacional e internacional (Larson y Ribbot 2004). Desde 1970 el concepto de sustentabilidad se ha convertido en un principio guía en el uso y manejo de los recursos naturales (WCED 1987), además se ha 
propuesto disminuir la distancia entre intereses y percepciones nacionales y locales, así como resolver los conflictos relacionado con recursos nacionales (Morales 2004). En este sentido, se puede relacionar con la descentralización.

La evolución del concepto sustentabilidad puede ubicarse desde dos orígenes: los movimientos sociales y las instituciones. El primero involucra diferentes movimientos sociales, los cuales desde su práctica diaria y su experiencia han cuestionado el modelo dominante de desarrollo, especialmente sus efectos socioambientales negativos. Las segundas son resultado de la presión realizada por estos movimientos sobre las instituciones formales para lograr un desarrollo sustentable local, por la definición e implementación de ciertas políticas públicas que se basan en la descentralización y el desarrollo local (ibíd.).

De acuerdo con las ideas de Martínez Allier (2004), estos movimientos pueden ser considerados representativos de un ambientalismo popular, porque surgen de la contradicción entre la conservación ecológica y el crecimiento económico. La expresión de estos movimientos se relaciona con la defensa del uso y manejo de los recursos naturales por estructuras comunitarias y locales, amenazando al mercado e incluso al Estado. Además, también pueden ser considerados como una reacción contra la degradación cultural y ambiental en el ámbito global. Según Leff (1998), el concepto de sustentabilidad ha emergido como un límite simbólico en la reorientación conceptual del proyecto dominante de civilización humanitaria.

El concepto de sustentabilidad implica una visión de largo plazo, considerando las relaciones sociedad-naturaleza en procesos de desarrollo (WCED 1987), que de acuerdo con Toledo (2000) busca una «modernidad alternativa». Esta modernidad alternativa, 
referida básicamente a un desarrollo más integral, se basa en nuevas éticas planetarias, solidarizándose con todos los miembros de la especie humana, y con todos los seres vivientes y elementos del planeta (Toledo 1999).

De lo anterior queda claro que el surgimiento del concepto de sustentabilidad puede ser comprendido como una reacción hacia los procesos dominantes de desarrollo, de manera que ha provocado diversas manifestaciones de resistencia contra las políticas de exclusión social. Leff (1998) menciona que estas expresiones de resistencia están articuladas con la construcción de un paradigma alternativo, en el cual los recursos endógenos surgen como potenciales viables para reconstruir los procesos económicos, y como parte de una nueva racionalidad productiva. Esta nueva racionalidad se caracteriza por una fuerte dimensión social y se basa también en la diversidad cultural, la democracia y las capacidades productivas de la naturaleza. Desde esta perspectiva, la relación entre lo global y lo local resulta ser fundamental para alcanzar la sustentabilidad y provee un marco referencial para la construcción de alternativas viables. De nuevo siguiendo a Leff (1998), la búsqueda por la sustentabilidad en el ámbito global es confrontada con el reto de generar estrategias que permitan la unión de localidades a escala global, partiendo de la autonomía cultural, identidades étnicas y condiciones ecológicas específicamente locales.

La relevancia de lo local en relación con la sustentabilidad local es también señalada por Touraine (1998), quien menciona que los movimientos sociales y las prácticas sociopolíticas innovadoras no están construidos inicialmente en el ámbito global o nacional. Al contrario, es en el espacio local donde las experiencias concretas conectadas a través de 
relaciones interpersonales se reúnen en prácticas sociales que confrontan los efectos negativos de la globalización económica (ver también, van der Ploeg 1992).

Aunque los movimientos sociales se caracterizan por una gran diversidad, también han tenido la capacidad de formular propuestas de desarrollo rural viables con potencial de convertirse en políticas para la solución de la pobreza y el manejo sustentable de los recursos. En algunos casos, estas propuestas han sido implementadas preferentemente separadas de los esfuerzos institucionales formales, como muestra nuestro caso de estudio. En otros casos, estos movimientos se han convertido en parte de las políticas formales (Caporal y Costabeber 2004). En otras palabras, los movimientos sociales locales han sido capaces de contribuir con elementos relevantes a las estrategias de desarrollo rural, así como han tenido la capacidad de negociar con instituciones formales (Morales 2004). Entre los elementos de sus diferentes propuestas se pueden mencionar el fortalecimiento de la agricultura familiar, la multifuncionalidad agropecuaria, acceso seguro a la tierra y los recursos naturales, promoción de la vida y cultura rural, sustentabilidad de las actividades de desarrollo rural, comercio justo, y gobierno local (ibíd.).

\section{EL CONTEXTO MEXICANO}

La presencia de un gobierno centralizado ha sido un factor constante en la historia mexicana. Constitucionalmente, el país es una República federal con 32 estados soberanos, y cada estado es compuesto por municipios independientes. Sin embargo, en la práctica el gobierno federal continúa teniendo una fuerte influencia sobre los gobiernos estatales y municipales. Durante los aproximadamente setenta años de gobierno continuo, 1934-2000, 
del Partido Revolucionario Institucional, PRI, el centralismo se ha acentuado. Además, el Estado federal a lo largo de todo este tiempo ha ocupado un mandato muy amplio en la economía, política y vida social mexicana.

En México, el proceso de descentralización es diverso, y recientemente los resultados han empezado a percibirse, siendo de naturaleza contradictoria. Por ejemplo, es posible identificar acciones institucionales incipientes orientadas hacia la entrega de responsabilidades a los gobiernos estatales y municipales, considerando el manejo de los recursos naturales y las políticas de desarrollo rural. Sin embargo, esta trasferencia de toma de decisión falta ser acompañada por recursos financieros adecuados, al no haberse desarrollado procesos pertinentes de descentralización fiscal y distributiva (Morales 2004).

Asimismo, algunos de los aspectos de descentralización han resultado ser acciones dirigidas hacia la privatización de recursos naturales. Como parte de estos procesos de privatización de facto, el Estado ha iniciado un proceso de trasformación legal institucional que inicia en 1992 con cambios en la Constitución, respecto al derecho de tierra de campesinos y comunidades indígenas. Este proceso de trasformación ha continuado con las reformas hechas a diferentes leyes, como la Ley Federal de Agua, la Ley de Desarrollo Forestal, y la reciente Ley de Bioseguridad, entre otras.

Aunque la descentralización de instituciones y políticas acompañantes es un paso importante hacia el impulso de un desarrollo local sustentable, ha faltado incorporar los múltiples actores locales en el proceso de toma de decisión y en la asignación de recursos. En otras palabras, todavía sigue ausente un proceso de apropiación por la sociedad civil que 
permita la construcción de una sociedad renovada, donde los actores locales controlan y deciden la definición de múltiples sendas de desarrollo.

Es por tal razón que los movimientos de la sociedad rural mexicana no solo han demandado la descentralización, sino también enfatizan la importancia de fortalecer el papel de las sociedades civiles en el proceso del desarrollo sustentable. En este sentido, las experiencias concretas de estos movimientos permiten un importante número de procesos de aprendizaje social relacionados con la descentralización y el empoderamiento (Morales 2004). No obstante, el aparato institucional mexicano y las políticas públicas continúan resistiéndose a estas demandas con la intención de defender sus espacios políticos en el campo.

El México contemporáneo tiene muchos problemas ambientales; situación especialmente grave, ya que México es considerado el quinto país con mayor biodiversidad en el mundo. Una de las causas de la crisis ecológica es el uso intensivo de la tecnología de la Revolución Verde, que ha llevado al deterioro de los ecosistemas en tasas irrefrenables (Warman 2001). Más específicamente, México ha sido afectado por la acelerada deforestación, erosión del suelo, contaminación del suelo y del agua, y la pérdida de la biodiversidad. La dimensión cultural de esta crisis se refleja en trasformaciones de las estructuras comunitarias y culturas rurales (Calva 2004).

La dimensión económica de la crisis se muestra en una pérdida de rentabilidad de muchas actividades agrícolas de los pequeños y medianos productores, que comprende $87 \%$ de los campesinos mexicanos. Como consecuencia, la pobreza existente en el campo 
mexicano hace que anualmente miles de mexicanos migren a las grandes ciudades —como Guadalajara o la ciudad de México—y a Estados Unidos de Norteamérica (ibíd.).

Las políticas públicas se basan en la promoción de un modelo que favorece la agricultura comercial; no considera la diversidad biológica o socioproductiva. En este sentido, un estudio realizado por Gómez Cruz et al. (2005) sobre las limitaciones institucionales para la agricultura orgánica en México muestra que 45\% de los campesinos entrevistados menciona la falta del apoyo gubernamental y los excesivos procedimientos burocráticos, $20 \%$ menciona un marco legal inapropiado y $15 \%$ de los campesinos indica que las políticas públicas incluso se oponen al fortalecimiento de la producción orgánica. En otras palabras, aquellos campesinos que no siguen el modelo dominante de desarrollo rural tienen un lugar limitado o nulo en las políticas de desarrollo rural.

\section{EL CONTEXTO JALISCIENSE}

El estado de Jalisco se ubica en el occidente de México. Su paisaje se caracteriza por una gran diversidad ecológica; existen distintos ecosistemas con diversos climas, topografía, vegetación y suelo. La agricultura tiene una larga historia en la zona y también se caracteriza por una diversidad cultural y productiva. La vida rural, junto con sus actividades

forestales y agrícolas, han sido a lo largo de la historia un componente central de la identidad cultural de los habitantes de Jalisco, y el sector rural juega un papel muy importante en la economía y la política del estado (Ochoa 2005).

El campo de Jalisco también soporta los altos costos sociales, culturales y ambientales del modelo neoliberal de desarrollo. Actualmente, el sector agropecuario se 
caracteriza por la larga crisis que afecta a todas las zonas rurales de México, a pesar de su relativo éxito productivo (Morales 2004). La emigración está desintegrando lentamente no solo a la familia y a la agricultura, también a las comunidades rurales y sus identidades culturales (Arroyo 1995). Además, la extensión de monocultivos y el modelo tecnológico ha llevado a un deterioro severo del suelo, el agua y la vegetación natural. El caso más reciente de los monocultivos es la expansión del cultivo de agave azul, que se ha expandido a nuevas regiones del estado de Jalisco a través de la agricultura de contrato, y donde ya se mostraron los costos ambientales (Martínez et al. 2007, Bowen y Gerritsen 2007).

\section{NUEVOS MOVIMIENTOS SOCIALES Y MANEJO DE RECURSOS NATURALES}

La búsqueda de alternativas locales de desarrollo desde la perspectiva de sustentabilidad rural se ha convertido en una tendencia global. Es en este contexto que se puede comprender el surgimiento de muchas organizaciones y redes de campesinos, indígenas, agricultores, consumidores, organizaciones no gubernamentales, centros de investigación y universidades en México.

Como se mencionó previamente, las políticas rurales en el campo mexicano pueden caracterizarse por una larga tradición de protección nacional y estatal, creando una dependencia política en los campesinos. Sin embargo, debido a la reciente crisis económica, las protestas se han convertido en una parte del discurso y la práctica en el campo. Así, similar a otras partes del mundo, un aumento en la politización del campo mexicano puede observarse en las últimas dos décadas del siglo veinte (Woods 2003). Movimientos como el Ejercito Zapatista de Liberación Nacional, El Barzón, El campo no 
aguanta más, o Sin maíz no hay país, están activamente intentando cambiar las políticas en las zonas rurales de México. Su misión consiste en el fortalecimiento de la economía de las familias rurales, la conservación de los recursos familiares, y el aumento de la capacidad de producción. La búsqueda de soluciones para vencer la crisis del campo mexicano está aumentando, y las experiencias de todo el país muestran la viabilidad de estrategias alternativas para el desarrollo rural. Toledo (2000) menciona más de dos mil comunidades rurales involucradas en estos procesos, muchas de las cuales se ubican en el centro y sur de México.

Un indicador relevante del crecimiento y viabilidad de estas estrategias es el aumento de la tierra dedicada a la agricultura orgánica en México. La agricultura orgánica ha aumentado de 23,265 en 1996 a 308,000 ha en 2005, y continúa aumentando. Asimismo, los productores orgánicos cultivan más de treinta productos diferentes, como café, vegetales, hierbas, y plantas aromáticas y medicinales. Además, la agricultura orgánica también ha aumentado el número de fuentes de empleo y la cantidad de dinero obtenido a través de la exportación, esta última alcanzó 270,000,000 de dólares en 2005. Otro hecho importante es que $85 \%$ de los productores orgánicos mexicanos poseen menos de un promedio de 30 ha de tierra (Gómez Cruz et al. 2005). Probablemente, los números reales y los productos son mayores, si tomamos en cuenta lo que Rist (2003) llama «la producción oculta de alimentos orgánicos». Con este término, se refiere a la producción agrícola y ganadera en sistemas campesinos principalmente tradicionales. Ellos bien pueden ser considerados orgánicos, debido al hecho de que se basan en prácticas agroecológicas tradicionales. 
Los movimientos campesinos mencionados pueden ser considerados o llamados «nuevos» movimientos sociales (Woods 2003) que, hablando generalmente, pueden caracterizarse como «surtido de grupos comprometidos en la acción política rural» (ibíd., p. 310). Sus luchas se pueden localizar en la defensa de estructuras locales — socioculturales, organizativas y económicas-, así como por el control sobre los diferentes campos de la vida diaria. En otras palabras, estos movimientos tienen como objetivo enfatizar la necesitad de enfoques endógenos hacia el desarrollo rural (Ray 1999, Long y van der Ploeg 1994), y van relacionados con la defensa de los estilos de vida específicos del sector rural (ibíd., Escobar 2001, Gerritsen et al. 2005, Gerritsen 2006). Es también en este contexto que surgen nuevas formas específicas del manejo de recursos naturales, como han revelado varios estudios (Posey 1999, Gerritsen, 2002, Malischke et al. 2005). Estas pueden ser consideradas nuevas en el sentido de que representan novedosas interacciones entre el contexto local y global.

En el estado de Jalisco la tendencia descrita anteriormente por buscar estrategias alternativas para el desarrollo rural sostenible también puede ser observada al ofrecer un gran número de experiencias locales importantes (Gerritsen et al. 2004). Estas experiencias incluyen pequeños empresarios —mujeres y hombres-, campesinos, organizaciones indígenas y grupos ecológicos urbanos, cuyas actividades consisten en agricultura sustentable, producción y comercialización de hierbas y plantas medicinales, turismo rural y educación ambiental, entre otras. Es también en esta coyuntura que surge la Red de Alternativas Sustentables Agropecuarias. 


\section{LA EXPERIENCIA DE LA RED DE ALTERNATIVAS SUSTENTABLES}

\section{AGROPECUARIAS}

La Red de Alternativas Sustentables Agropecuarias, RASA, se origina como una iniciativa fuera del contexto institucional formal, que sigue la tendencia global y nacional hacia la construcción de patrones alternativos de desarrollo local y sustentable. En el contexto estatal de Jalisco, caracterizado por la exclusión social de los actores sociales en áreas rurales marginados por las instancias de gobierno, RASA debe ser comprendida como una iniciativa en la búsqueda por nuevas formas de participación ciudadana en la definición de toma de decisión y estrategias de desarrollo. Estas experiencias tienen poca relación con las instituciones gubernamentales, y RASA ha buscado contribuir, sobre todo, al fortalecimiento del papel de la sociedad civil en la definición e implementación de políticas públicas, y en el desarrollo de nuevas relaciones entre servidores sociales y el entorno social. El trabajo de RASA se une a otros esfuerzos dedicados a vencer la crisis social, ecológica, económica y cultural, que afectan en México a los campesinos, indígenas, agricultores y actores urbanos marginados.

\section{CARACTERIZACIÓN GENERAL DE LA RED}

Actualmente RASA está conformada por veinte grupos de campesinos, indígenas, mujeres y consumidores urbanos, y numerosas organizaciones no gubernamentales, universidades e individuos. El trabajo de RASA comenzó en 1999 con siete comunidades rurales, en diferentes partes del estado de Jalisco. Cada uno de estos grupos ya había tenido experiencias alternativas de desarrollo, pero no fue hasta 1999 que comenzaron a 
articularse entre sí. Aunque las diferentes experiencias se basaban en distintas perspectivas ideológicas y metodológicas, ciertos elementos eran puntos comunes, lo que permitió esta articulación. Los puntos en común son: los esfuerzos tomados para fortalecer la agricultura multifuncional, para mantener la vida e identidad rural, y el contribuir en la solución a la pobreza. Más aún, todos los grupos forman parte de la perspectiva sustentable y tienen relación con movimientos sociales en México y América Latina. La Red se origina del intercambio de experiencias de estos diferentes grupos y emerge como un espacio local donde un modelo alternativo de desarrollo rural es construido colectivamente.

Desde 1999 el movimiento ha experimentado un crecimiento significativo en el número de familias interesadas en la agricultura sustentable, lo que ha generado un aumento en la demanda de asistencia técnica (Figura 1, Tabla 1).

En función de alcanzar su misión, actualmente RASA se enfoca en tres áreas de atención relacionadas: formación para la agricultura sustentable, asesoría y acompañamiento para la agricultura sustentable, y comercio justo de productos agroecológicos. 
Figura 1. Municipios con presencia de grupos de la RASA

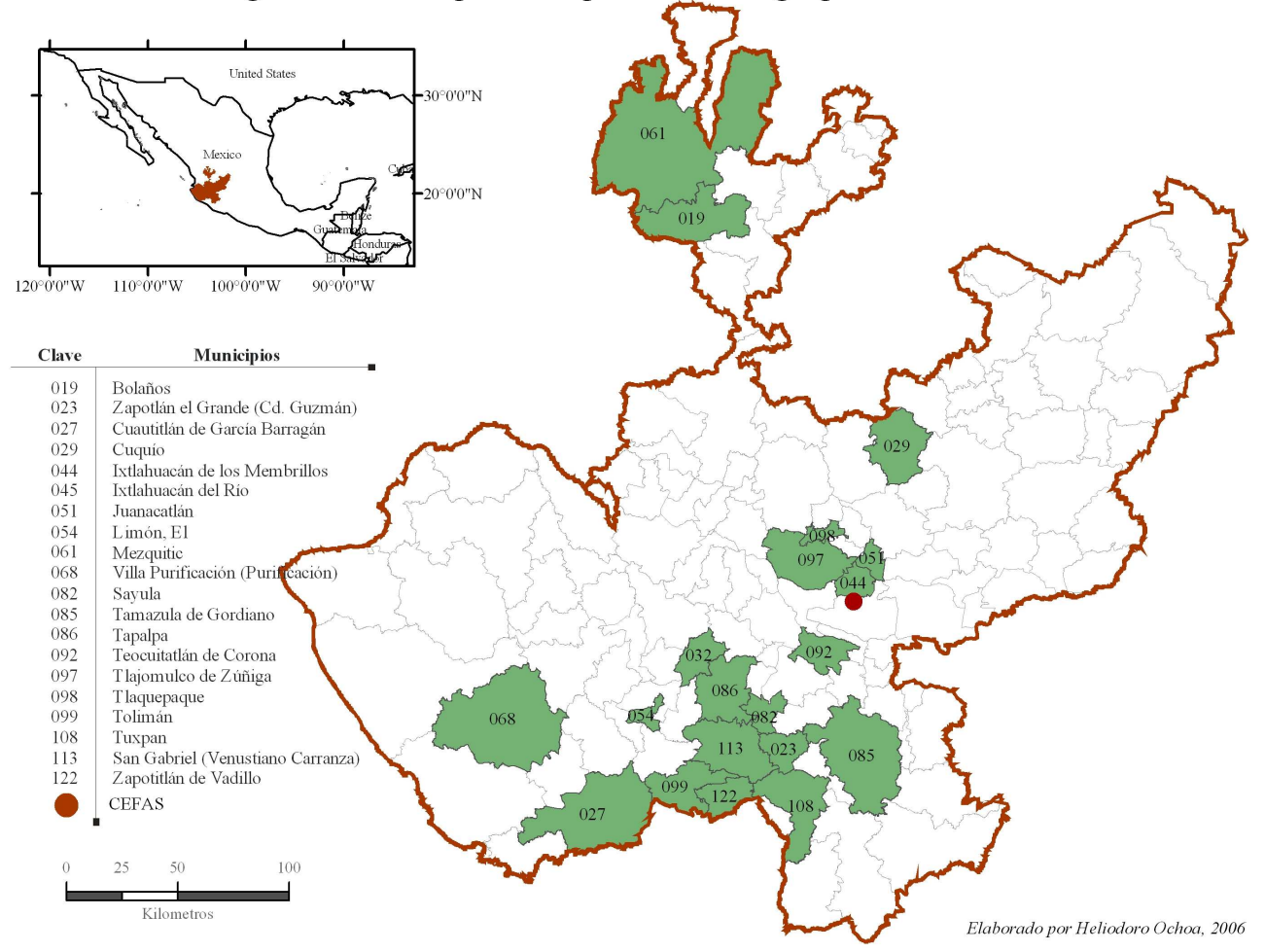


Tabla 1: Una vista general de los diferentes actores participando en la RASA

\begin{tabular}{|c|c|c|c|c|}
\hline $\begin{array}{l}\text { Tipo de } \\
\text { Organización }\end{array}$ & $\begin{array}{l}\text { Número total de } \\
\text { organizaciones }\end{array}$ & $\begin{array}{l}\text { Número total de } \\
\text { familias } \\
\text { involucradas }\end{array}$ & $\begin{array}{l}\text { Número total de } \\
\text { personas } \\
\text { involucradas }\end{array}$ & Comentarios \\
\hline $\begin{array}{l}\text { Grupos (locales) } \\
\text { campesinos }\end{array}$ & 20 & 200 & 800 & $\begin{array}{l}\text { Diferentes tipos de productores } \\
\text { (orientados a la subsistencia y al } \\
\text { mercado) }\end{array}$ \\
\hline Asesores & 6 & & 30 & $\begin{array}{l}\text { De diferentes instituciones: ONG'S, } \\
\text { univesidades, consultorias privadas y } \\
\text { organizaciones locales }\end{array}$ \\
\hline $\begin{array}{l}\text { Grupos de } \\
\text { consumidores }\end{array}$ & 6 & 25 & 100 & $\begin{array}{l}\text { Consumidores urbanos interesados en } \\
\text { productos orgánicos y comercio justo }\end{array}$ \\
\hline Otros & Variable & Variable & Variable & $\begin{array}{l}\text { Esto es variable, debido al hecho de } \\
\text { que la mayoría de los encuentros de la } \\
\text { RASA son abiertos al público general. }\end{array}$ \\
\hline
\end{tabular}

Fuente: Elaboración propia.

\section{MARCO CONCEPTUAL DE RASA}

En el sector rural existen amplias regiones, especialmente en comunidades campesinas e indígenas donde las actividades agropecuarias y forestales — la agricultura en su acepción amplia — son parte fundamental en la economía y en la vida de los habitantes rurales. En esas regiones el diseño y puesta en práctica del desarrollo rural sustentable requiere estrategias de producción coherentes con esta perspectiva alternativa, y un elemento importante en esta discusión es la noción de agricultura sustentable (Morales 2004).

La agricultura sustentable, de acuerdo con la propuesta de Gliessman (2002), comprende los siguientes componentes: una menor dependencia de los insumos externos; la seguridad y autosuficiencia alimentaria; los procesos de autogestión y participación 
comunitaria; el uso de recursos renovables locales; el mantenimiento de la capacidad productiva; el respeto a la diversidad cultural; impactos benignos sobre el medio ambiente; el uso de la experiencia y conocimiento local; el mejoramiento de la diversidad biológica, y la atención a los mercados locales y externos. Estos componentes presentes en el concepto de Gliessman muestran los puntos a enfatizar dentro de las estrategias para la agricultura sustentable, y evidencian también las diferencias con el enfoque de la agricultura industrial. La propuesta es útil además para definir parámetros de sustentabilidad en los procesos de desarrollo rural.

La agricultura sustentable, siguiendo a Menezes (1995), implica los siguientes elementos: el énfasis en la agricultura familiar y en una agricultura diversificada, orientadas hacia la seguridad alimentaria, con reformas agrarias que mejoren las condiciones de vida en el medio rural, soportadas por políticas agrarias de crédito en extensión e investigación, con participación campesina y ciudadana, que fiscalicen las agriculturas ecológicamente nocivas. Esta idea presenta una concepción amplia de la agricultura sustentable, y precisa sus dimensiones sociales y políticas, asimismo deja claro que la agricultura sustentable es mucho más que un enfoque tecnológico y se ubica como elemento de procesos de desarrollo alternativos.

Una de las características de la agricultura sustentable es su multifuncionalidad, concepto ya presente en políticas públicas de varios países, sobre todos europeos. La multifuncionalidad asume que la agricultura cumple funciones que no son mercantiles —como las ecológicas, sociales y culturales—, y reconoce el fracaso de la industrialización de la agricultura. Se trata de una pequeña revolución frente al neoliberalismo y sus 
promotores, y la multifuncionalidad es un camino para ir hacia una agricultura sustentable, más respetuosa con los seres humanos, con los animales, con el ambiente (Bové y Dufour 2001).

Los efectos del modelo neoliberal no solamente se dan en el ámbito productivo, también se reflejan en las cadenas productivas y los mercados. Por la creciente competencia con productos convencionales e inestabilidad de los precios en los mercados, los productos resultados de la agricultura sustentable están en constante incertidumbre por la falta de protección en los diferentes mercados.

Ante tal riesgo de la producción y la falta de mercados alternativos, el comercio justo lejos de ser un reto es ya una necesidad planteada desde los mismos actores rurales en proceso de desarrollo local sustentable, y se manifiesta específicamente en cada experiencia durante el paso de la agricultura convencional a la agricultura sustentable. Una vez que se han satisfecho las necesidades de consumo familiar y se generan excedentes en la producción se buscan caminos distintos de comercialización regidos por fundamentos que buscan beneficiar directamente a los pequeños productores, crear organizaciones autónomas y democráticas, tomar en cuenta a los consumidores locales e instaurar precios justos, y sobre todo que estos procesos vayan encauzados hacia el desarrollo local sustentable (Bernardo 2007).

El comercio justo se puede considerar también como un movimiento ciudadano que está buscando dar salida a los productos ecológicos desde otros caminos, apoyando así la segunda fase de las estrategias sustentables locales, ya que cuando surge la necesidad de vender da cuenta de un proceso de segundo nivel, pasando del autoconsumo y la 
autosuficiencia alimentaría al complemento económico familiar caracterizado por la venta de excedentes en la producción. Una vez que aparece la necesidad de vender en un proceso, se debe generar el medio propicio para fomentar entre los distintos actores involucrados formas de mercados alternativos para no truncar su proceso y la economía local (Roozen y van der Hoff 2002).

\section{EL ENFOQUE METODOLÓGICO}

Los diferentes actores sociales e instituciones que participan en RASA emplean diferentes perspectivas y enfoques hacia el trabajo en las diferentes comunidades rurales. Hay grupos que se dedican más a la producción, mientras otros dan mayor importancia a la comercialización. Asimismo, algunos producen más hortalizas, mientras otras siembran y industrializan plantas medicinales, por ejemplo. Si bien RASA se caracteriza por una diversidad de miembros, existe un consenso sobre las bases generales metodológicas, siendo las principales la agroecológica y la educación popular.

La primera es una de las orientaciones teóricas que se incluye en la construcción del desarrollo rural desde un punto de vista alternativo, y es generada como una respuesta al reto del desarrollo sustentable (Guzmán et al. 2000). La contribución de la agroecología a RASA es la integración de los cuestionamientos sociales y ambientales, favoreciendo un enfoque más integrado en el diseño y evaluaciones participativas de tecnologías alternativas. De esta forma, las estrategias de RASA contemplan no solo el aumento de la producción de las cosechas sino también la conservación de los recursos naturales y el fortalecimiento de la identidad comunal. Por otro lado, el énfasis que la agroecología y su 
metodología dejan en el conocimiento de pequeños campesinos ha sido un gran beneficio para las acciones de RASA. El conocimiento local y la experimentación autóctona de tecnologías alternativas son considerados un punto de partida central. De esta manera, la confianza de los productores participantes en su propio conocimiento y tecnologías es fortalecida, entre otras cosas, debido al hecho de que ellos reconocen la viabilidad y productividad de su propio trabajo. Un caso muy concreto son los abonos orgánicos, que han permitido mejorar la calidad de los suelos y terminar con el intermediarismo económico.

La educación popular parte de un punto de vista epistemológico en el cual los procesos educativos son considerados una forma de diálogo entre los actores partiendo de sus palabras comunes. De acuerdo con el pedagogo brasileño Paulo Freire, padre fundador de esta metodología: «nadie dice la palabra solo. Decir algo significa decirla para otros. Por eso la verdadera educación es diálogo. Y este encuentro [por ejemplo, el diálogo] no puede darse en el vacío, sino que se da en situaciones concretas, de orden social, económico, político» (1985: 16). Entonces, el reconocimiento de que todo actor actúa de acuerdo con su realidad, su visión, y establecer el diálogo en esta realidad, implica que «nadie educa a nadie, los seres humanos se educan entre sí». En otras palabras, todos los actores son capaces de aprender, la falta de esto es consecuencia de las condiciones objetivas en las que se encuentra (ibíd.).

La estrategia de la educación popular pone gran énfasis en las metodologías alternativas de trabajo con poblaciones rurales y está orientada hacia la labor de los asesores agrícolas con un diálogo horizontal y continuo con los campesinos. Esto permite la 
revaloración del conocimiento local al igual que un cambio hacia las prácticas rurales de educación, orientadas no solo hacia la aceptación no crítica de la modernización rural, sino de igual manera hacia la trasformación de la realidad rural. La educación popular ha sido tomada en cuenta en tres orientaciones: la investigación participativa, el diálogo entre los diferentes sistemas de conocimiento, y el enfoque de campesino a campesino.

\section{LA ESTRUCTURA ORGANIZATIVA}

La estructura organizativa de RASA ha evolucionado a través de los años de acuerdo con su crecimiento como red; es decir, con los diferentes actores sociales e instituciones que son miembro. Por una parte, siempre ha tenido la intención de mantener una estructura flexible, en función de ser capaz de responder rápidamente a cuestiones operativas. Por otro lado, la estructura ha sido pensada para promover y fortalecer la participación, horizontal y democrática. Como resultado de los procesos de planeación estratégica y de las experiencias obtenidas a lo largo de los años, RASA ha desarrollado una estructura que puede caracterizarse por ser horizontal, democrática y de naturaleza participativa. A través del tiempo, la Red ha estado definiendo su estructura funcional actual alrededor de cuatro elementos principales.

- Los Grupos Locales, siendo diferentes grupos campesinos en el estado de Jalisco, generalmente acompañados por al menos un asesor profesional.

- La Asamblea General es la máxima autoridad, la cual está integrada por todos los miembros de RASA: hombres y mujeres campesinos e indígenas, así como asesores 
- El Consejo Campesino lo componen ocho miembros campesinos de diferentes regiones del estado de Jalisco, que son elegidos por los miembros de la asamblea general. El consejo campesino indica las líneas estratégicas generales y las consideraciones a largo plazo.

- El Consejo Operativo establece y monitorea los planes anuales de operación y da seguimiento a las decisiones tomadas por la asamblea general y el consejo campesino. Está integrado por cinco asesores y un campesino, y es elegido por todos los miembros de la asamblea general.

Figura 2. Estructura Organizativa de la RASA

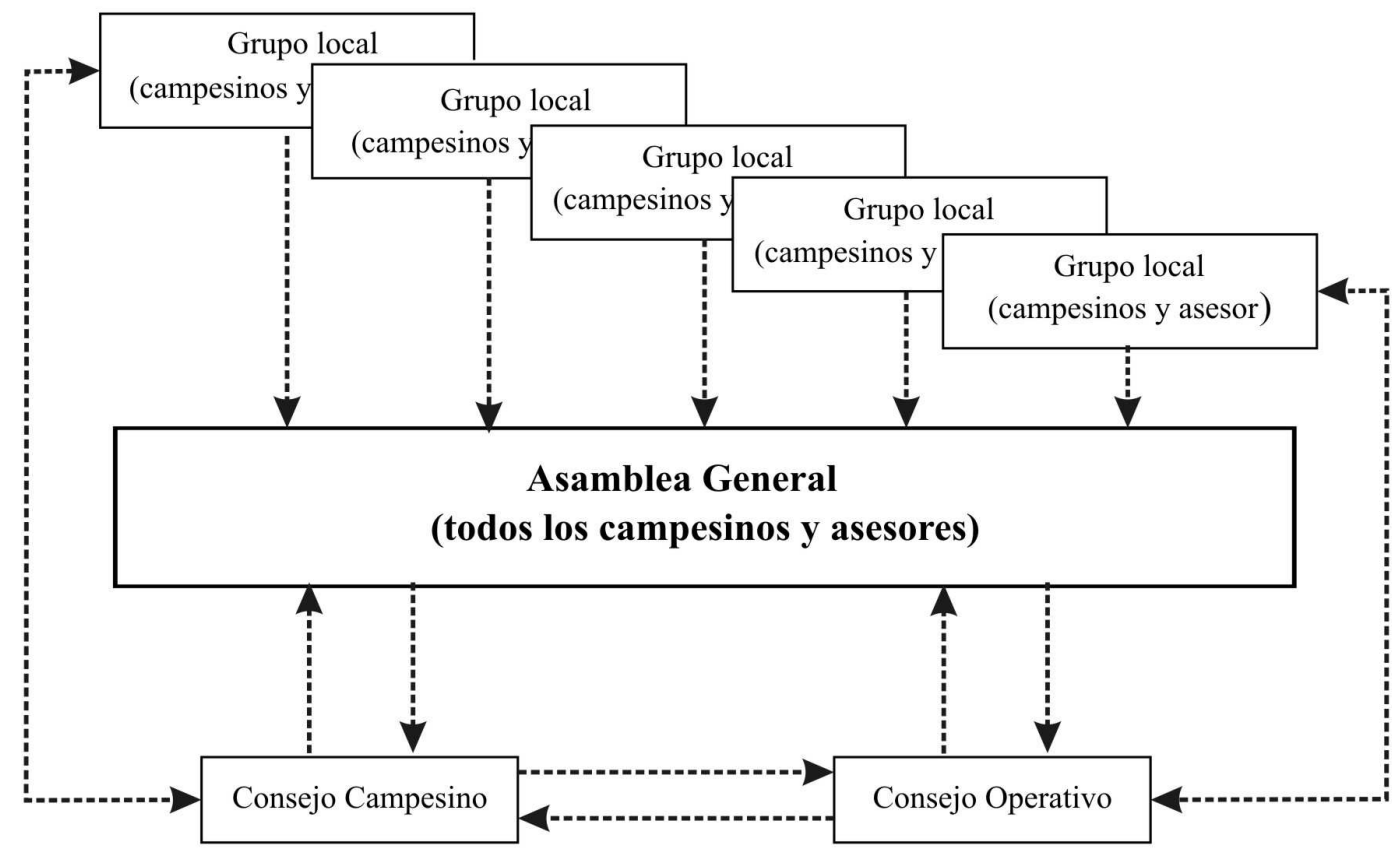

Fuente: Elaboración propia. 
Tabla 2. Frecuencia de las reuniones con la RASA

\begin{tabular}{|l|c|l|}
\hline Tipo de grupo & Reuniones Anuales & Comentarios \\
\hline Grupos locales & $12-16$ & Variable de acuerdo con el grupo específico \\
\hline Asamblea general & 5 & $1-2$ reuniones de planeación 3-4 encuentros campesinos \\
\hline Consejo Campesino & $3-6$ & $1-2$ reuniones con el Consejo Operativo \\
\hline Consejo Operativo & $12-16$ & $1-2$ reuniones con el Consejo Campesino \\
\hline
\end{tabular}

Fuente: Elaboración propia.

Los horizontes de RASA han crecido a través de la incorporación de diferentes grupos de indígenas, mujeres, campesinos y consumidores. Este crecimiento ha provocado que el asunto de género sea una de las propuestas y actividades de la red. De esta manera, la participación de RASA también ha evolucionado de atender cuestiones tecnológicas y productivas a la inclusión de las demandas de las mujeres rurales, y ha avanzado hacia la integración de la visión compleja de la familia campesina como un punto de referencia para las acciones de sustentabilidad. De hecho, el número de grupos de mujeres campesinas se ha incrementado de uno a seis, y también aumenta la presencia de asesoras en los organismos de dirección de RASA, donde dos de ellas participan como miembros del Consejo Operativo, quienes han sido responsables de algunas de las acciones fundamentales, como el comercio justo, entre otras.

\section{ETAPAS EN EL DESARROLLO DE LA RED}

Si bien RASA tiene relativamente poco tiempo de existencia, en su evolución de manera general es posible reconocer tres etapas. La primera comienza con su creación en 1999 hasta 2001. En este periodo, el énfasis principal de la Red fue la de experimentar con técnicas de agricultura sustentable para mejorar la subsistencia y la autonomía alimentaria 
de los miembros participantes. En este periodo RASA también dirigió sus esfuerzos a incorporar nuevos grupos en la red. La segunda etapa corresponde al periodo entre 2001 y 2003. Durante el mismo, las acciones continuaron siendo la formación a campesinos y la capacitación técnica sobre agricultura orgánica para grupos existentes y nuevos; sin embargo, RASA extendió su base social al incorporar el trabajo de género. Este enfoque de género se orientó hacia las familias rurales, vivienda sustentable y trasformación de los productos alimentarios. Tales acciones han estado más relacionadas con el espacio de acción de las mujeres.

La tercera y actual etapa en el desarrollo de RASA comenzó en 2003, cuando el comercio justo de los productos de los miembros de la Red obtuvo un papel muy importante en las actividades. Actualmente, un total de 125 productos son comercializados en distintos puntos de venta en el estado de Jalisco, con un énfasis en Guadalajara y la ciudad de México. Las actividades de comercio justo han permitido, sobre todo, establecer y reforzar la relación entre productores rurales y consumidores urbanos, especialmente en la capital del estado de Jalisco, Guadalajara. A partir de esta experiencia RASA se ha vinculado al esfuerzo de la Red de Tianguis Orgánicos en México.

Además, es durante este año que el mandato de RASA se expandió de un énfasis productivo hacia asuntos más generales sobre sustentabilidad y conservación, como los debates sobre servicios ambientales, conservación del agua y el bosque, y el tema de las semillas genéticamente modificadas. Finalmente, ha sido también en los últimos dos años que RASA ha buscado activamente la articulación con otros movimientos sociales en Jalisco y la ciudad de México. 


\section{APRENDIZAJES}

Se pueden encontrar varios aprendizajes en el trabajo de RASA. A pesar del contexto adverso para la agricultura familiar en México, los diferentes grupos que integran la Red han aumentado exitosamente sus experiencias «aprendiendo en la práctica». Esta forma de trabajar ha mostrado su viabilidad a lo largo del tiempo en términos productivos, ecológicos y económicos. Desde esta perspectiva, RASA ahora tiene la capacidad de diseñar y operar propuestas tecnológicas basadas en prácticas y métodos ya evaluados en las condiciones locales. Esto ha provocado un crecimiento en la Red, y desde 1999 el número de grupos casi se ha triplicado, como ya se mencionó. Adicionalmente, se puede observar un aumento continuo de nuevos campesinos y asesores en las reuniones y talleres de RASA. Además la Red se ha convertido en un punto de referencia sobre agricultura orgánica y comercio justo, y ahora es un actor social importante en el estado. Finalmente, RASA ha participado en la formación y capacitación a técnicos y asesores de Desarrollo Rural y de Desarrollo Humano, a través de cursos y seminarios sobre desarrollo local y sustentabilidad. Sin embargo, las relaciones con agencias gubernamentales continúan siendo complicadas y dependen en gran parte de los funcionarios involucrados.

Otro aprendizaje en la experiencia de RASA indica la viabilidad de las trasformaciones rurales basadas en un enfoque de campesino a campesino. Durante los últimos ocho años, los campesinos de la Red han aumentado su conocimiento sobre agricultura orgánica y han adquirido un papel relevante en la formación de otros campesinos de nuevo ingreso. 
Actualmente la Red cuenta con un grupo de diez campesinos y campesinas con varios años de experiencia en agricultura orgánica que actúan como formadores en los talleres y reuniones y cuyo proceso ha sido analizado en detalle por Bernardo (2007). Estos formadores también dirigen sus esfuerzos a compartir sus experiencias y conocimiento con otros grupos y organizaciones. Es en este contexto que RASA, con el apoyo de organizaciones de Suiza, España y México, ha iniciado la construcción de un centro de formación sobre agricultura orgánica cerca de Guadalajara, con el objetivo de fortalecer las capacidades de los campesinos. Este centro se termino en el 2007 y actualmente está operando permanentemente.

Otro aprendizaje se refiere a la disponibilidad de recursos externos que han caracterizado el trabajo de RASA desde su comienzo. El desarrollo de la Red se ha basado principalmente en las posibilidades financieras de sus miembros. Sin embargo, esto no ha sido un inconveniente para organizar actividades y obtener resultados satisfactorios. De hecho, han sido estos resultados los que han atraído algunos recursos económicos externos.

Para concluir, el trabajo de RASA y los resultados que se han alcanzado se deben a los esfuerzos colectivos de todos sus miembros. Las contribuciones de los participantes en términos de trabajo, materiales — didácticos- y recursos, son operados mediante dos puntos comunes: fortalecimiento de una alternativa de desarrollo local a través del trabajo con productos orgánicos y las actividades de comercio justo. Es este pensamiento lo que ha permitido que RASA se mantenga independiente de las agencias gubernamentales, y ha fortalecido la autonomía de sus miembros y sus acciones. 


\section{RETOS}

En el contexto nacional y global, y desde las diferentes experiencias de RASA, se pueden identificar varios retos, de los cuales queremos mencionar cuatro. El primero se relaciona con la marginación del campo mexicano y jalisciense, que provoca la emigración y el abandono del campo. Mientras grandes cantidades de jóvenes campesinos abandonan sus comunidades en busca de mejores oportunidades, las actividades del campo son responsabilidad de adultos con poca participación de campesinos jóvenes (cf. Warman 2001). Es por esta razón que la mayoría de los campesinos de RASA son adultos, y que la presencia de gente joven es limitada, poniendo en riesgo la futura viabilidad de la Red. Enfrentados a esta situación, la Red tiene un reto importante, la formación en producción ecológica y las alternativas de comercio justo deben ser lo suficientemente atractivas para la gente joven en las zonas rurales. Con ello, la Red debe poder ofrecer posibilidades viables que les permitan mantener el arraigo en sus comunidades de los campesinos. En otras palabras, debe permitirles vivir con dignidad, preservar sus recursos naturales y fortalecer su identidad cultural y comunitaria. El aumento en la presencia de campesinos jóvenes en RASA facilitará la difusión de experiencias y permitirá incrementar los niveles de participación campesina en el manejo y operación de la red.

Un segundo reto refiere a su relación con diferentes movimientos en los ámbitos regional, nacional y global, que también buscan alternativas sustentables para el desarrollo rural. Por ahora, la Red ha establecido diversas relaciones con diferentes actores sociales. A escala regional se ha articulado con organizaciones ciudadanas y grupos sociales, agrupados en torno al Consejo Impulsor y Regulador de la Producción Orgánica en Jalisco, 
CIRPRO, mientras que en el nacional RASA está apoyando esfuerzos de comunidades indígenas en Chiapas y Veracruz a través de cursos específicos y capacitación en agricultura orgánica y comercio justo. En el ámbito global RASA forma parte del Movimiento de Agricultura Ecológica Latinoamericano, MAELA. Sin embargo, estas actividades pueden fortalecerse y de esta manera generar un movimiento social alternativo global (Morales 2004).

Un tercer reto se relaciona con el comercio justo y la conexión con nuevos movimientos sociales urbanos; por una parte, un número importante de las familias participantes busca alcanzar la autosuficiencia y mejorar su nivel nutricional. No obstante, también hay numerosos campesinos que intentan combinar el autoabasto con actividades de comercio justo, lo que enfatiza la necesidad de desarrollar nuevos canales de comercialización. RASA decidió activamente orientar las actividades de comercio justo hacia consumidores urbanos, con quienes sea posible iniciar un proceso de conocimiento y diálogo mutuo que facilite establecer relaciones basadas en el comercio justo.

Finalmente, queremos mencionar el cuarto reto, siendo éste la relación de la Red con instituciones de gobierno. Es claro que uno de los éxitos de RASA ha sido su autonomía e independencia del Estado y los partidos políticos, y nuestra visión del futuro contempla fortalecer esta capacidad de autogestión. Sin embargo, las propuestas de RASA también están orientadas hacia la participación activa en la discusión y definición de nuevas políticas públicas para el fortalecimiento de la sustentabilidad en la agricultura familiar de Jalisco. Este reto se refiere a la articulación de la perspectiva actual de autonomía e independencia de las instituciones estatales con la participación activa de RASA en el 
diseño e implementación de políticas públicas. En este contexto, es importante mencionar de nuevo la incapacidad de las agencias del gobierno estatal para establecer relaciones de trabajo propias y complementarias con las organizaciones sociales, como RASA. Desde la perspectiva del Estado, RASA es concebida como un novedad en el diseño de nuevas políticas públicas y como elemento contracorriente en las políticas públicas actuales. Más aún, las acciones de miembros de la Red para establecer relaciones equitativas y respetuosas con las agencias gubernamentales se han encontrado con servidores públicos que no están acostumbrados a que grupos de campesinos tengan su propia idea del modelo de desarrollo a seguir.

\section{DISCUSIÓN Y CONCLUSIÓN}

En este artículo describimos la experiencia de RASA en el contexto de la descentralización, los movimientos sociales y el manejo de los recursos naturales. Esta descripción muestra que el trabajo de la Red va más allá de una mera capacitación de los campesinos en técnicas orgánicas de producción y en la promoción del comercio justo. Se considera también la acción colectiva para dirigir asuntos sociales y políticos. Es por eso que pensamos que RASA puede ser considerada como un nuevo movimiento social (Woods 2003, Rist 2003), cuyas características son la acción descentralizada y el liderazgo múltiple y colectivo. La acción colectiva de RASA va más allá de los objetivos productivos, y puede ser caracterizada como informal y espontánea, aun cuando un proceso institucional se ha comenzado en aras de responder al mejoramiento de las necesidades y demandas de los diferentes actores involucrados en la Red. 
Desde la perspectiva de RASA, su más amplio mandato tiene que ver con la aspiración de alcanzar mayor equidad en las relaciones entre el campo y la ciudad, donde la cultura y conocimiento rural tengan un lugar y sean reconocidos. Para el futuro, RASA aspira contribuir en procesos de trasformación social que parten desde las culturas campesinas e indígenas, y atender temas como justicia y equidad social y ambiental, entre otros. Es por eso que los miembros de RASA conciben la Red en el sentido de generar, promover y articular formas sustentables de producción familiar y comunitaria, que se alcanzará al apoyar procesos autónomos sociales considerados como alternativas al discurso dominante y prácticas del desarrollo rural.

Siguiendo a Della Porte y Diani (1999, citado en Woods 2003), la evolución de la agenda de RASA para las acciones colectivas puede tipificarse por ser modificada de metas específicas, de tipo operativo, a más generales, de tipo estratégico; lo último permite una mejor articulación con otros movimientos sociales (véase Kaltoft 2001). Además, se puede caracterizar por una naturaleza más segmentada, que es entrelazada por los grupos locales, la asamblea general, el consejo campesino y el consejo operativo. En este sentido, el proceso de institucionalización de RASA probablemente sea uno de los mayores retos de los siguientes años; se tendrá que encontrar un equilibrio que permita la articulación horizontal y democrática, y la participación con una acción colectiva. También, las acciones colectivas de RASA pueden ser categorizadas al reflejar ambos sentidos rurales, el «progresivo»y el «aspirativo» (Woods 2003: 318). Siguiendo la descripción de Woods, de estas dos formas de ruralismo el progresivo surge de la resistencia de los miembros de RASA ante las prácticas agroindustriales o prácticas de agricultura moderna, al igual que 
ante varios aspectos negativos de la globalización. Con respecto al aspirativo, el debate actual sobre los cultivos genéticamente modificados es la expresión más reciente de esta forma de ruralismo. Las acciones colectivas de RASA pueden ser consideradas como una forma de aspiración al ruralismo, mientras los miembros mantengan fuertemente y defiendan su bagaje e identidad cultural.

Es importante notar aquí que RASA ha surgido desde la sociedad civil en el campo de Jalisco, donde su contacto con las instituciones estatales ha sido muy limitado y algunas veces problemático, como ya mencionamos en varias ocasiones. Esto lleva a la idea de que además de la descentralización democrática y administrativa se puede identificar un tercer tipo de descentralización que se caracteriza por los grupos de la sociedad civil uniendo fuerzas al margen de la descentralización. En otras palabras, los esfuerzos formales de descentralización no fortalecen este tipo de experiencias, más bien crean nuevos espacios políticos para acciones colectivas y permite que los movimientos sociales salgan a la superficie.

Los esfuerzos de descentralización y globalización de las naciones, Estados y compañías trasnacionales, se pueden considerar altamente antidemocráticos y potencialmente destructivos para los nuevos movimientos sociales (Larson y Ribbot 2004), como RASA. La privatización de los recursos hidrológicos y la biodiversidad puede considerarse el siguiente paso en la expansión de la globalización económica (Barlow y Clarke 2002). Al mismo tiempo, las compañías trasnacionales están aumentando e interrumpiendo las estructuras locales sociales con la introducción de las tecnologías de la 
Revolución Verde en el pasado y la biotecnología en el presente (Morales 2004, Rubio 2004).

Finalmente, la experiencia de RASA ilustra el surgimiento desde abajo de un incipiente movimiento social, cuyas características están formadas por las capacidades de los campesinos y asesores, las ideologías, las historias políticas y sociales, y las formas de organización social. En otras palabras, su efectividad se basa en la construcción de propiedades endógenas de la población del campo en México (Toledo 2000, Gerritsen 2006). Estas propiedades endógenas son multidimensionales y multifuncionales; incorporan esos elementos exógenos siempre y cuando dichos elementos permitan fortalecer las estrategias locales (Van der Ploeg et al. 2002, Escobar 2001, Gerritsen et al. 2004). Es necesario enfatizar que estos esfuerzos están altamente relacionados con una postura ideológica y con una visión particular sobre el desarrollo del campo en particular y el desarrollo de la sociedad en general (Gerritsen et al. 2004, 2005; Gerritsen y Morales 2001, cf. Ray 1999). Además están relacionadas con la naturaleza de la descentralización y los espacios políticos ofrecidos por el Estado. 


\section{BIBLIOGRAFÍA}

Arrighi, Giovanni, 1999, El largo siglo XX. Dinero y poder en los origines de nuestra época. Cuestiones de Antagonismo. AKAL, Madrid.

Arroyo A., Jesús (comp.), 1995, Y ante todo la población rural persiste. Efectos de la modernización económica en el campo mexicano. Universidad de Guadalajara, CUCEA, Guadalajara.

Barlow, Maude y Tony Clarke, 2002, Blue gold. The fight to stop the corporate theft of the world's water. The New Press, New York, London.

Beck, Ulrich, 1992, The Risk Society. Towards a new modernity. SAGE publications, London, Thousand Oaks, New Delhi.

Bernardo, María de Jesús, 2007, «Estrategias de formación en desarrollo rural sustentable con actores sociales: El caso de la Red de Alternativas Sustentables Agropecuarias de Jalisco». Tesis de maestría. Universidad Internacional de Andalucía, España.

Bové, José y Francois Dufour, 2001, El mundo no es una mercancía: los agricultores contra la comida basura. Editorial Icaria, España.

Bowen, Sarah y Peter R. W. Gerritsen, 2007, «Reverse leasing and power dynamics among agave farmers in western Mexico». Agricultura and Human Values, núm. 24, pp. 473-488. 
Calva, José Luis, 2004, «Ajuste Estructural y TLCAN: efectos en la agricultura mexicana y reflexiones sobre el ALCA». El Cotidiano, año 19, núm. 124, pp. 14-22.

Caporal, Franscisco y José Costabeber, 2004, Agroecología e extensao rural. Contribuicoes para a promocao do desnvolvimento rural sustentavel. MDA, SAF, DATER-IICA, Brasil, D. F.

Della Porta, Donatella y Mario Diani, 1999, Social movements: an introduction. Blackwell, Oxford.

Escobar, Arturo, 2001, «Culture sits in places: reflections on globalism and subaltern strategies of localization». Political Geography, núm. 20, pp. 139-174.

Fraga, Gabino, 2000, Derecho administrativo. Editorial Porrúa, México.

Freire, Paulo, 1985, La educación como práctica de la libertad. Siglo XXI editores, México.

Gerritsen, Peter, 2002, Diversity at stake. A farmers' perspective on biodiversity and conservation in Western Mexico. Wageningen Studies on Heterogeneity and Relocalisation, núm. 4. Wageningen University, The Netherlands, Wageningen.

2004, «Productos regionales y la sustentabilidad». Ponencia presentada en el Curso Internacional en Producción Agropecuaria, organizado por ITESO, SEDER, CIRPRO y RASA, 25 a 30 de octubre de 2004. 
2006, «On endogenous rural development and new images of rurality in western Mexico». Ponencia presentada en el XI Congreso Internacional de LASA Association, marzo 15-18, mecanuscrito. San Juan, Puerto Rico.

Gerritsen, Peter, Víctor Villalvazo, Pedro Figueroa y Gerardo Cruz, 2005, «Fortaleciendo procesos endógenos en la Costa Sur de Jalisco: Imágenes de una nueva ruralidad emergente». Ponencia presentada en el I Foro Académico Interinstitucional Diálogos sobre el Sur de Jalisco: Actualidad y Futuro del Desarrollo, 22 de febrero, mecanuscrito. Zapotlán el Grande, Jalisco, México.

Gerritsen, Peter, Gerardo Cruz, Víctor Villalvazo, y Pedro Figueroa, 2004, «"Productos regionales" en el Occidente de México: ¿respuestas locales frente a la globalización económica?». Ponencia presentada en el congreso Internacional Agroempresas rurales y Territorio, ARTE, 1-4 de diciembre, mecanuscrito. Toluca, Estado de México.

Gerritsen, Peter, Jaime Morales H., 2001, «Conservación de la biodiversidad. El papel del desarrollo y la participación local. Sociedades rurales. Producción y medio ambiente». vol. 2, núm. 2, pp. 87-95, diciembre.

Gliessman, Stephen, 2002, Agroecología: procesos ecológicos en agricultura sustentable. Coediciones de Agruco, Maela, CATIE, GTZ, UAY, Gobierno de Tabasco y Universidad de California, Costa Rica. 
Gómez Cruz, Manuel Ángel, Rita Schwentesius, y Laura Gómez Tovar, 2005, Agricultura, Apicultura y Ganadería Orgánica en México 2005: Situación, Retos y Tendencias. CONACYT, SAGARPA, UACH, Chapingo, México.

Guzmán C., Gloria, Manuel González de Molina y Eduardo Sevilla G., 2000, Introducción a la agroecología como desarrollo rural sostenible. Ediciones Mundi-Prensa, Madrid, Barcelona, México.

Kaltoft, Pernille, 2001, «Organic farming in the late modernity: at the frontier of modernity or opposing modernity?». Sociologia Ruralis, vol. 41, núm. 1, pp. 146-158, enero.

Larson Anne y Jesse Ribot, 2004, «Democratic decentralisation through a Natural Resource Lens: An Introduction». En Democratic Decentralisation through a Natural Resource Lens. Experiences from Africa, Asia and Latin America, Ribot J. C. y A. M. Larson (eds.). The European Journal of Development Research, vol. 16, núm. 1, spring. Taylor and Francis Ltd., Abingdon, United Kingdom.

Leff, Enrique, 1998, Saber Ambiental. Siglo XXI, México.

Long, Ann y Jan Douwe van der Ploeg (eds.), 1994, Born from within. Practice and perspective of endogenous rural development. Van Gorcum Publisher, Assen.

Malischke,' Therese, F. Rodolfo González y Peter Gerritsen, 2005, «Percepciones campesinas sobre la degradación ambiental Una comparación de agricultores orgánicos y convencionales en el ejido de La Ciénega, municipio de El Limón, 
Jalisco, México». Ponencia presentada en V Congreso de AMER, 25-28 mayo, mecanuscrito. Oaxaca, México.

Martínez Alier, Joan 2004, El ecologismo de los pobres: Conflictos ambientales y lenguajes de valoración. Editorial Icaria, Barcelona.

Martínez Rivera, Luis Manuel, Peter Gerritsen, Juan Jesús Rosales Adame, Arturo Moreno H., Sarahy Contreras M., Arturo Solís M., Luis Eugenio Rivera C., Óscar Cárdenas H., Luis Ignacio Íñiguez D., Ramón Cuevas G., Carlos Palomera G., Edith García R., Ángel Aguirre G. y José Luis Olguín L., 2007, en prensa, «Implicaciones socioambientales de la expansión del cultivo de agave azul en el municipio de Tonaya, Jalisco». En Agaves de importancia económica en México, Colunga-García Marín et al. (eds.). CICY, Academia Mexicana de Ciencias.

Menezes, Francisco, 1995, «Agricultura sustentable y pobreza rural». En Agroecología y desarrollo sustentable, memorias del 2. ${ }^{\circ}$ seminario internacional de Agroecología. Ediciones de la Universidad Autónoma Chapingo, México.

Morales Hernández, Jaime, 2004, Sociedades rurales y naturaleza. En busca de alternativas hacia la sustentabilidad. ITESO, Universidad Iberoamericana, Guadalajara.

Ochoa G., Heliodoro, 2005, «Agricultura, sociedad y espacios productivos en el sur de Jalisco». Tesis de maestría. Universidad Iberoamericana de Puebla, Puebla. 
Posey, Darryll, 1999, Cultural and spiritual values of biodiversity. A complementary contribution to the Global Biodiversity Assessment. Intermediate Technology Publications, London.

Ray, Cristobal, 1999, «Endogenous development in the era of reflexive modernity». Journal of Rural Studies, vol. 15, núm. 3, pp. 257-267.

Rist, Stephan, 2003, «Organic Agriculture as Social Movement. Re-thinking sustainable agriculture in developing countries». En Food Matter: Food Security and Soils, Lahmar, R., M. Held y L. Montanarella, pp. 108-114. Torba Soil \& Society, Montpellier.

Roozen, Nico y Frans van der Hoff, 2002, La aventura del comercio justo. Una alternativa de globalización; por los fundadores de Max Havelaar. UCIRI, Editorial El Atajo, México.

Rubio, Julio (coord.), 2004, Alimentos transgénicos, ciencia, ambiente y mercado: un debate abierto. UNAM, Siglo XXI, México.

Toledo, Víctor, 1999, «Hacia una modernidad alternativa». Revista Renglones, núm. 41-42. Instituto Tecnológico y de Estudios Superiores de Occidente, Tlaquepaque, México

2000, La paz en Chiapas: ecología, luchas indígenas y modernidad alternativa. UNAM, Quinto Sol, México.

Touraine Alain, 1998, ¿Podremos vivir juntos? Fondo de Cultura Económica, México. 
Van der Ploeg, Jan Douwe, 1992, «The reconstitution of locality: technology and labour in modern agriculture». En Labour and locality: uneven development and the rural labour process», Marsden, T., R. Lowe y S. Whatmore (eds.), pp. 19-43. David Fulton Publishers, London. (Critical perspectives on rural change series, IV.)

Van der Ploeg, Jan Douwe, Ann Long y Jo Banks, 2002, Living countrysides. Rural development processes in Europe: the state of the art. Elsevier, Doetinchem.

Warman, Arturo, 2001, El campo mexicano en el siglo XX. Fondo de Cultura Económica, México.

Woods, Michael, 2003, «Deconstructing rural protest: the emergence of a new social movement». Journal of Rural Studies, núm. 19, pp. 309-325.

World Comission on Environment and Development, WCED, 1987, Our common future. Oxford University Press, Oxford.

Fecha de recepción: 05 de febrero de 2008

Fecha de aceptación: 03 de mayo de 2008 